\title{
EchoGéo
}

18 | 2011

Varia

\section{Entretien avec Étienne Verkindt}

\section{Anne Hertzog}

\section{OpenEdition}

Journals

Édition électronique

URL : https://journals.openedition.org/echogeo/12823

DOI : 10.4000/echogeo.12823

ISSN : 1963-1197

\section{Éditeur}

Pôle de recherche pour l'organisation et la diffusion de l'information géographique (CNRS UMR 8586)

Référence électronique

Anne Hertzog, « Entretien avec Étienne Verkindt », EchoGéo [En ligne], 18 | 2011, mis en ligne le 05 décembre 2011, consulté le 31 juillet 2021. URL : http://journals.openedition.org/echogeo/12823 ; DOI : https://doi.org/10.4000/echogeo.12823

Ce document a été généré automatiquement le 31 juillet 2021.

EchoGéo est mis à disposition selon les termes de la licence Creative Commons Attribution - Pas d'Utilisation Commerciale - Pas de Modification 4.0 International (CC BY-NC-ND) 


\title{
Entretien avec Étienne Verkindt
}

\author{
Anne Hertzog
}

1 Étienne Verkindt est titulaire d'un master de géographie soutenu à Lille 1. Il est aujourd'hui doctorant, en contrat CIFRE avec le Conseil Général du Nord où il est recruté pour le montage et le suivi des projets culturels européens portant sur la valorisation transfrontalière des patrimoines, tout en débutant une thèse consacrée aux relations entre paysage et mémoire.

2 - Anne Hertzog (AH). Quelle est votre formation universitaire?

3 - Étienne Verkindt (EV). J'ai fait une licence de sciences humaines et sociales, mention Géographie et aménagement «Géographie et Dynamiques des Territoires » (Lille 1), puis une maîtrise en sciences et technologie, mention Aménagement Urbanisme et Développement des Territoires, Parcours « Géographie, Milieux, Territoires » (Lille 1). J'ai poursuivi par un master 2 en Aménagement Urbanisme et Développement des Territoires, spécialité « Tourisme-Loisirs-Sport » (Lille 1). Mon mémoire de M1 portait sur Les représentations du monde et de la frontière dans les romans de Jean Christophe Grangé (dir. P. Picouet). Un autre, réalisé collectivement en M2, questionnait la possibilité de l'écotourisme en Nord-Pas de Calais. Sans être spécialisé dans les questions patrimoniales, ce master professionnel évoquait largement le patrimoine à travers un certain nombre de cours, de travaux de terrain et d'études. Par exemple, le diagnostic (collectif) réalisé en M2 sur le développement touristique du Pays de St Omer incluait le patrimoine, notamment parce qu'il prenait en compte certains sites patrimoniaux tels que l'ascenseur à bateau des Fontinettes à Arques (Pas-de-Calais). Dans ce cadre, je me suis formé à la méthodologie de projet, notamment sur des sites patrimoniaux.

4 - AH. Comment en êtes vous arrivé à travailler sur les questions patrimoniales, notamment sur les sites mémoriels liés aux conflits mondiaux dans le département du Nord?

5 - EV. Dans le cadre de l'année de M2 Pro, j'ai effectué un stage de six mois au sein du Conseil général du Nord, qui avait sollicité ma directrice de master afin de recruter un étudiant pour travailler sur le projet Interreg IVa (France Wallonie Vlanderen) «Transmussites 14-45». Une action intitulée «Structurer et valoriser le paysage des 
guerres : de la Flandre au Pas-de-Calais ») était spécifiquement consacrée à l'étude et la valorisation des paysages de mémoire des guerres de 14/18 et 39/45.

Il s'agit d'un projet transfrontalier lancé en 2008 qui couvre le département du Nord, du Pas-de-Calais et la province de Flandre occidentale et dont l'objectif est la valorisation du patrimoine de mémoire et la mise en réseaux des musées et sites des deux guerres mondiales. J'ai été recruté pour participer à la réflexion sur la notion de paysage de mémoire, qui en était à ses débuts, puisqu'elle est apparue avec ce programme Interreg. L'idée première était que les paysages avaient joué un rôle important durant la première guerre mondiale : cours d'eau, topographie etc... Ils sont aujourd'hui toujours perçus et pensés comme le dernier témoignage des guerres.

7 - AH. Il y a donc eu un rapprochement des trois territoires dans le cadre des projets Interreg sur la valorisation des « paysages de mémoire »?

8 - EV. En Flandre occidentale (Belgique), les acteurs de la province travaillaient depuis quelque temps déjà sur la gestion, la sauvegarde et la valorisation de ce patrimoine paysager, notamment par l'intermédiaire du réseau « Guerre et Paix ». Aussi, s'étaientils montrés particulièrement réactifs et volontaires dans ce domaine. C'est d'ailleurs la province de Flandre qui porte toujours l'action sur les paysages de mémoire en collaboration avec les départements du Nord et du Pas de Calais.

9 Pour le département du Nord, le $90^{\mathrm{e}}$ anniversaire de la Grande Guerre a suscité une prise de conscience de l'intérêt patrimonial des sites liés à la Première Guerre mondiale. Les projets européens ont cependant particulièrement accentué ces préoccupations mémorielles et patrimoniales, de même que la perspective du centenaire du premier conflit mondial. Certes, il existait déjà des structures qui s'intéressaient à cette thématique auparavant (associations, archives...) mais cette dernière a été réellement renforcée (au niveau institutionnel dans le département du Nord) par le développement des projets culturels européens. Deux projets européens, dont le département du Nord est chef de file, portent ainsi sur ce patrimoine dans le cadre d'Interreg IVa : « Mémoire de la Grande Guerre » et « Transmussites 14-45 ».

10 - AH. Quelles méthodes et quelle démarche avez-vous privilégié, en tant que géographe, dans le cadre de ce projet culturel européen orienté vers la valorisation du patrimoine lié aux deux guerres mondiales?

11 - EV. Lorsque je suis arrivé, il s'agissait vraiment de " débroussailler » le terrain! Je suis arrivé au moment de l'inauguration du projet! J'ai alors été chargé de voir ce qui se faisait dans les autres collectivités pour mettre en place une méthodologie d'étude et d'inventaire des paysages mémoriels de la Première Guerre mondiale.

12 Afin de réaliser l'action consacrée aux paysages de mémoire dans le cadre de «Transmussites 14-45», Nous avons constitué un groupe de travail en 2009 avec des représentants des trois territoires : la province de Flandre occidentale, le Nord, et le Pas-de-Calais. Le groupe de travail transfrontalier était (et est toujours) composé de la chargée de mission « Mémoire » du Pas de Calais (de formation plutôt historienne), du coordinateur du réseau «Guerre et Paix » de la province de Flandre occidentale (archéologue), et de moi-même en qualité de géographe. En 2009, nous formions le «noyau dur ", tout en impliquant d'autres structures en fonction des aspects abordés : le service régional de l'Inventaire, le Comité Régional du Tourisme qui développait en même temps une politique de mémoire etc. L'action entreprise implique une forte et essentielle démarche collective, en lien avec les universités. C'est ainsi que l'Université 
de Gand a été missionnée pour la réalisation d'une étude transfrontalière des paysages de mémoire à partir de la lecture et de l'analyse d'outils bien identifiés : les SIG, les photographies aériennes et les cartes anciennes. Nous avons décidé ensemble d'une méthodologie d'étude et d'inventaire des paysages comme par exemple étudier les paysages avant, après la guerre et aujourd'hui. Un gros travail de cartographie a été mené à partir d'un travail de terrain et d'une importante recension photographique. Nous bénéficions d'expériences antérieures dans le département du Pas-de-Calais avec notamment l'analyse des sites du "verrou de Souchez ", une lecture croisée paysagère et historique que le conseil général du Pas-de-Calais souhaitait étendre à l'ensemble de la zone de front. Ces premiers travaux ont constitué pour nous une base méthodologique certaine. Certes, notre travail a également dû s'adapter aux approches différentes des collectivités, d'autant que les paysages étaient aussi sensiblement différents selon les territoires.

13 - AH. Comment la géographie théorique ou conceptuelle est-elle mobilisée dans ce travail en réponse à une demande institutionnelle?

14 - EV. Mon mémoire de master 2 intitulé Etude préliminaire sur la gestion et la valorisation des paysages de mémoire des deux guerres mondiales, réalisé en étroite collaboration avec mon chef de service de l'époque, Freddy Dolphin, qui avait beaucoup travaillé sur le projet Interreg IVa Septentrion dont la thématique concernait les villes fortifiés, développait un volet théorique et un volet plus pratique. Pour le Conseil général, l'approche théorique des paysages, qui m'occupait beaucoup dans le cadre du mémoire de master, était un élément intéressant mais n'était pas le but premier de mon recrutement. Il s'agissait avant tout de monter une méthodologie d'inventaire puis d'étude des paysages de mémoire. Cependant mon travail sur la notion de paysages de mémoire a permis d'identifier des problèmes de définition. De ce point de vue, mon profil de géographe était intéressant dès lors qu'il pouvait se construire avec d'autres savoirs déjà présents dans la structure. Les personnes attachées aux collectivités territoriales appartiennent a des champs disciplinaires différents (économie, sciences sociales, histoire, etc.), ont eu des parcours différents, tout particulièrement dans les services en charge de projets culturels, et c'est la une richesse tout à fait remarquable !

15 - AH. Quels liens peut-on établir entre cette réflexion et la formation théorique reçue en géographie?

16 - EV. J'avais travaillé sur les représentations du monde et des frontières dans les romans de Grangé. Deux aspects m'intéressaient particulièrement : la question de la frontière et celle des paysages. L'objectif était de montrer que le roman pouvait participer à la représentation des territoires et à la connaissance géographique. Il s'agissait d'utiliser le roman comme outil géographique. Ma méthodologie portait sur la manière dont un roman pouvait être lu de manière géographique, comment il pouvait participer à la création de représentations du monde. C'est vraiment ce travail qui m’a conduit à « ma » spécialité en géographie, à la fois sur la question de la frontière et sur la question des représentations, notamment par les travaux de mon directeur de master (P. Picouet) puis de mes directeurs de thèse actuels (P. Picouet et E. Glon). C'est également à ce moment là que j'ai mieux découvert l'importance de l'approche épistémologique en géographie, la réflexion sur la pensée géographique. Tout cela a indéniablement nourri la réflexion plus tardive sur la notion de paysage.

17 - AH. Quelle est votre situation actuelle, travaillez vous sur d'autres projets européens de valorisation des patrimoines? 
18 Après le stage de master, j'ai effectué un contrat de 4 mois au Conseil général qui m'a recruté car l'intérêt patrimonial pour les paysages de mémoire va croissant, notamment avec le lancement récent du processus de leur inscription au patrimoine mondial de l'Unesco! À certains égard, cette éventualité croise les projets déjà en cours, notamment "Tansmussites 14-45 ", mais aussi d'autres projets départementaux comme la mise en place de « cahiers de sites et de territoires » dont je suis chargé et qui constitueront des bases de réflexion sur la gestion et la valorisation des paysages de mémoire dans le cadre des territoires locaux.

19 Je suis aujourd'hui en contrat CIFRE avec le Conseil général du Nord. La convention réunit trois parties: l'étudiant, l'université avec un laboratoire de recherche - dans mon cas, le laboratoire Territoires, Villes Environnement et Société de Lille 1 - et le Conseil général du Nord. Je suis donc en thèse tout en travaillant pour le Conseil général, qui me permet de consacrer $50 \%$ de mon temps de travail à ma recherche. Mon sujet sur le lien entre mémoire et territoire - qui visiblement a intéressé tous les partenaires de la convention! - a été validé en 2011 (Association Nationale de la Recherche et de la Technologie).

$20 \mathrm{Au}$ sein du Conseil général du Nord, je suis donc toujours en charge, avec d'autres, du suivi des projets culturels européens et notamment de l'action paysage de mémoire du projet « Tansmussites 14-45». Durant mon stage, j'avais acquis certaines compétences que le conseil général a souhaité conserver. Celles-ci servent d'ailleurs dans ma participation au montage d'autres projets européens transfrontaliers dans le domaine du patrimoine. Par exemple, le programme transfrontalier «Murailles et jardins", programme de gestion écologique des villes fortifiées, dont le département du Nord est également chef de file, qui réunit 23 partenaires européens dans le cadre du programme Interreg des deux mers 2011.

21 - AH. Le contrat CIFRE permet de revenir sur la place du jeune chercheur en géographie dans la politique patrimoniale d'une collectivité territoriale...

22 - EV. Dans le cadre des projets européens j'effectue des missions financées en partie par les Fonds Européens de Développement Régional. Mais je garde bien à l'esprit que tout en travaillant sur des thématiques communes aux projets européens et aux recherches menées avec l'Université notamment sur les paysages de mémoire, je dois prendre en compte la complexité propre à la recherche académique tout en étant dans la réponse à la demande institutionnelle. Je me retrouve bien dans le contrat CIFRE car ce qui est intéressant, c'est de sortir du cadre strictement scientifique tout en faisant en sorte que la recherche scientifique apporte quelque chose à la collectivité et inversement.

23 Cela nous renvoie aux finalités de la géographie : à quoi sert la géographie ? Comment établir une relation entre la recherche académique et la recherche professionnelle ? ... Par exemple, sur le plan théorique, j'étudie le lien entre paysage et mémoire, moins travaillé que celui qui relie paysage et patrimoine. Le paysage est considéré et étudié comme un objet patrimonial depuis quelques décennies déjà en géographie. Dans le cadre de ma thèse qui n'en n'est qu'à ses débuts, je compte montrer en quoi la mémoire, dans son ancrage territorial, estaussi un objet géographique. La composante paysagère me paraît très importante mais il est clair que le recours par exemple à l'histoire et à la psychologie sociale me semble tout a fait essentiel. Articuler la réflexion théorique sur l'espace et les paysages à la démarche de projet peut s'avérer tout à fait intéressant tant pour la recherche universitaire que pour répondre à la demande institutionnelle. Toutefois il est important de noter que l'apport n'est pas 
unilatéral car il s'agit bien de mettre en place une relation entre recherche universitaire et non universitaire. La convention CIFRE permet d'être une sorte d'intermédiaire entre le monde académique et la Cité !

La devise du département du Nord est "Des Hommes et des Territoires ». Lorsqu'on travaille dans les collectivités, le territoire est une dimension essentielle, nécessaire et systématique. Les géographes peuvent donc à la fois intervenir dans sa gestion, et dans son développement; le géographe pense développement territorial, ce qui correspond bien à la demande des collectivités territoriales. C'est également l'approche qu'il va privilégier lorsqu'il s'intéresse aux questions patrimoniales.

\section{BIBLIOGRAPHIE}

Verkindt E., 2008. La représentation du monde et des frontières dans les romans de J.C. Grangé : "Le vol des cigognes", "Le concile de pierre", "La ligne noire". Villeneuve d'Ascq : Université de Lille 1, dir. P. Picouet, $124 \mathrm{p}$.

Verkindt E., 2009. (Etude préliminaire en vue de) la sauvegarde et de la mise en valeur des paysages de mémoire des guerres mondiales dans le département du Nord. Villeneuve d'Ascq : Université de Lille 1/ Conseil général du Nord.

Verkindt E., en cours. Mémoire et Territoire. Héritages de la première guerre mondiale dans les paysages des territoires du Nord de la France. Thèse de doctorat sous la direction de Picouet P. et Glon E. 2011- en cours.

\section{AUTEUR}

\section{ANNE HERTZOG}

Anne Hertzog, hertzog.anne@wanadoo.fr, est Maître de conférences à l'Université de Cergy

Pontoise. 